\title{
Étude de la spéciation de l'uranium en milieu biologique par électrophorèse capillaire et spectrofluorimétrie laser à résolution temporelle
}

\author{
S. SCAPOLAN*, E. ANSOBORLO*, C. MOULIN**, C. MADIC***
}

(Manuscrit reçu le 17 mars 1997, révisé le 7 juin 1997, accepté le 20 octobre 1997)

RÉSUMÉ La connaissance du devenir chimique de l'uranium en milieu biologique réclame des études plus approfondies pour comprendre le comportement de l'uranium dans l'organisme. La caractérisation des différentes formes complexées de l'ion uranyle peut être améliorée en combinant deux techniques : la spectrofluorimétrie laser à résolution temporelle (SLRT) et l'électrophorèse capillaire (EC). En effet, l'EC, adoptant le mode de focalisation isoélectrique (CIEF), permet la séparation des complexes suivant leurs points isoélectriques (pI) tandis que la SLRT est une méthode de spéciation à bas niveau qui rend possible l'identification des complexes de l'ion uranyle au moyen de la résolution temporelle et de la déconvolution spectrale. Les résultats qui concernent aussi bien les systèmes inorganiques (phosphate, bicarbonate) que les systèmes biologiques (citrate, transférine) sont présentés et discutés. La complexation uranium-transférine est mise en évidence par l'utilisation de l'EC en mode CIEF.

ABSTRACT Uranium speciation studies in biological medium by capillary electrophoresis and time-resolved laser-induced fluorescence.

The knowledge of the chemical state of uranium in biological medium requires more detailed investigations to understand the behaviour of uranium in the organism. The characterization of the different complexes of uranium can be improved by combining two techniques: time-resolved laser-induced fluorescence (TRLIF) and capillary electrophoresis (CF). CE, indeed, using the isoelectric focusing mode (CIEF), allows for the separation of the different complexes as a function of their isoelectric points (pI) and TRLIF as a speciation method leads to the identification at very low level of different uranyl complexes by temporal resolution and spectral unfolding. Results obtained on various inorganic chemical systems (phosphate, bicarbonate) together with biological systems (citrate, transferrin) will be presented and discussed. The complexation between uranium and human transferrin has been pointed out through CIEF.

\footnotetext{
* Institut de Protection et de Sûreté Nucléaire, département de protection de la santé de l'homme et de dosimétrie, service de dosimétrie, IPSN, BP. 38, 26701 Pierrelatte Cedex. France.

** CEA/DCC/DPE/SPEA, Laboratoire de Spectroscopie Laser Analytique, CE/SACLAY, 91191 Gif sur Yvette, France.

*** CEA/DCC/DRRV/SEMP, CEA/VALRHO, Marcoule, BP 171, 30207 Bagnols sur Ceze Cedex, France.
} 


\section{Introduction}

La contamination par les composés uranifères induit une toxicité radiologique et chimique. La recherche de thérapeutiques efficaces par l'action d'agents décorporants est souhaitable pour permettre l'élimination d'une contamination accidentelle. Dans ce cadre, les études de spéciation relatives aux interactions entre le radionucléide et les constituants biologiques demeurent primordiales (Scapolan et al., à paraître). Le but de ces études est, dans un premier temps, de déterminer les formes chimiques des complexes formés entre l'uranium et les composants sanguins, et dans un second temps, rechercher les ligands appropriés permettant de déplacer les équilibres de complexation au niveau sanguin et de diminuer la fixation sur les organes cibles pour accélérer l'excrétion des radiocontaminants.

Les données de la littérature indiquent qu'après son incorporation (principalement par l'inhalation, l'ingestion ou la blessure), l'uranium circule dans le sang sous la forme d'ion uranyle $\mathrm{UO}_{2}^{2+}$ (état d'oxydation VI) et est principalement complexé aux bicarbonates et citrates $(50 \%)$, aux protéines $(30 \%)$ et aux érythrocytes (20\%) (Chevari et Likhner, 1968; Pasquier et Bourguignon, 1977; Voetglin et Hodge, 1953). Ces études sont toutefois incomplètes pour permettre de comprendre le comportement chimique de l'ion uranyle en milieu sanguin.

Dans le cadre du présent travail, la spectrofluorimétrie laser à résolution temporelle (SLRT) et l'électrophorèse capillaire ont été développées en tant qu'outils analytiques spécifiques pour apporter de nouvelles données à ces études de spéciation. La SLRT, utilisée pour l'analyse élémentaire à très bas niveau des actinides et des lanthanides (limite de détection pour l'uranium inférieure au $\mathrm{ng} / \mathrm{L}$ ), permet la spéciation par le biais des données extraites du spectre de fluorescence (déplacement et intensité associés aux interactions), et des informations temporelles fournies par les temps de vie de fluorescence (caractéristique de l'environnement de la molécule). Des études antérieures avaient été centrées sur l'ion uranyle dans des milieux tels que le milieu hydroxo (Moulin et al., 1995), nitrique (Couston et al., 1995), organique ou en présence de surfactant (Moulin et al.; 1991; 1993; 1992).

Les présents résultats obtenus en SLRT concernent aussi bien des matrices inorganiques, avec des ligands tels que les carbonates ou les phosphates, que des milieux dits biologiques avec des ligands comme les citrates ou la transférine (protéine qui semble être un des principaux ligands de l'uranium dans le sang (Stevens et al., 1972)).

Dans le but de compléter les données obtenues concernant les interactions uranium-transférine, une étude complémentaire spécifique a également été menée au moyen de l'électrophorèse capillaire adoptant le mode focalisation isoélectrique (CIEF). L'électrophorèse capillaire est une technique analytique qui permet la séparation et l'identification des complexes métal-ligand. Le 
mode focalisation isoélectrique dans des capillaires (CIEF) autorise de rapides séparations de protéines et est employé (Kilar et Hjertén, 1989) pour l'analyse des formes moléculaires de la transférine, comprenant l'apotransférine ainsi que ses formes mono- et diferriques. La structure et la réactivité de la transférine étant décrites par ailleurs (Baker, 1994), l'objectif de cette étude est de mettre en évidence, par l'utilisation du mode CIEF, la liaison uranium-transférine.

\section{Matériels et méthodes}

\subsection{Instrumentation}

Un système d'électrophorèse capillaire P/ACE 5510 (Beckman, France) équipé d'un détecteur à barrettes de diodes et d'un logiciel Gold (version 8.10 ) ainsi que des capillaires de silice vierge sont utilisées pour les études en CIEF.

Pour la SLRT, un laser Nd-YAG opérant à 266 ou $355 \mathrm{~nm}$ et délivrant une énergie d'environ $3 \mathrm{~mJ}$ en $10 \mathrm{~ns}$ avec une fréquence de 10 à $20 \mathrm{~Hz}$ est utilisé en tant que source d'excitation. Le faisceau laser est collecté par un analyseur multicanal intensifié pulsé (FLUO 2001, DILOR, FRANCE) permettant la résolution temporelle du signal de fluorescence. Le temps d'intégration varie de 1 à $99 \mathrm{~s}$, permettant ainsi de faire varier la sensibilité de détection. L'électronique autorise la mise en place de portes de mesures (délai : de 0,1 à $99 \mu$ s et longueur de la porte : de 1 à $999 \mu \mathrm{s}$ ).

\subsection{Réactifs}

Des réactifs de haute pureté chimique et de l'eau désionisée ultrafiltrée sont utilisés pour toutes les expérimentations.

\subsubsection{SLRT}

Systèmes uranium - hydroxyde, phosphate, citrate et bicarbonate - Les solutions d'uranium VI en milieu perchlorate de sodium $0,1 \mathrm{M}$ (Aldrich) sont obtenues par dilution d'une solution mère préparée par dissolution d'uranium métal ultra-pur dans de l'acide perchlorique $60 \%$ (Fluka). Les solutions de bicarbonate, phosphate et de citrate sont réalisées, respectivement, par dissolution de $\mathrm{NaHCO}_{3}, \mathrm{Na}_{2} \mathrm{HPO}_{4}$ et d'acide citrique en milieu $\mathrm{NaClO}_{4}$ $0,1 \mathrm{M}$. Les $\mathrm{pH}$ sont ajustés par ajouts de $\mathrm{HClO}_{4}$ ou de $\mathrm{NaOH}$.

Systèmes uranium - transférine - La solution d'uranium VI $\left(4 \times 10^{-5} \mathrm{M}\right.$ $\mathrm{pH}=3$ ) est préparée par dissolution de nitrate d'uranyle hexahydraté (Merck) en milieu perchlorate de sodium $10^{-3} \mathrm{M}$. La solution d'apotransférine $10^{-6} \mathrm{M}$ est obtenue par dissolution d'apotransférine (Aldrich) dans de 
l'HEPES $10^{-4} \mathrm{M}$ (acide $\mathrm{N}$-(2-hydroxyéthyl)-pipérazine-N'-2-éthanesulfonique) en présence de bicarbonate $\left(10^{-4} \mathrm{M}\right)$ et est ajustée au $\mathrm{pH}=7,4$ par ajouts de $\mathrm{NaOH}$.

\subsection{2. $C I E F$}

La solution d'apotransférine à $10 \mathrm{mg} / \mathrm{ml}$ est préparée dans un tampon HEPES $20 \mathrm{mM}$ contenant $20 \mathrm{mM}$ de $\mathrm{NaHCO}_{3}$. Le pH est fixé à 7,4 par de la soude (solution a). Pour les études des complexes uranium-transférine, $50 \mu \mathrm{l}$ d'une solution de nitrate d'uranyle $10 \mathrm{mM}\left(10^{-3} \mathrm{M} \mathrm{HCl}\right)$ est ajoutée à $500 \mu \mathrm{l}$ de la solution a (solution $\mathrm{b}$ ). Les solutions $\mathrm{a}$ et $\mathrm{b}$ sont incubées à température ambiante durant une heure et sont ensuite diluées au dixième dans une solution à $2 \%$ de Bio-Lyte $5 / 7$ avant injections.

\subsection{Procédures}

\subsubsection{SLRT}

Les études en SLRT qui concernent les systèmes uranium-bicarbonate, phosphate et citrate sont menées à partir d'un $\mathrm{pH}$ acide $(\mathrm{pH}=2-3)$; ce $\mathrm{pH}$ est ensuite régulièrement augmenté par des additions de soude. La concentration d'uranium est fixée à $2,8 \times 10^{-7} \mathrm{M}$ et différents rapports molaires $\mathrm{U}$ : ligand sont effectués (l'ultime rapport molaire correspond à la concentration du ligand en milieu sanguin) :

- $1: 0 ; 1: 1 ; 1: 10 ; 1: 100$ et $1: 89285$ pour le système U:bicarbonate,

- $1: 0 ; 1: 1 ; 1: 100$ et $1: 3750$ pour le système $U: p h o s p h a t e$,

- et $1: 0 ; 1: 1$ et $1: 568$ pour l'étude uranium-citrate.

Pour les études du système uranium-transférine, une solution d'uranium $\left(4 \times 10^{-5} \mathrm{M}-\mathrm{pH}=3\right)$ est successivement ajoutée à une solution d'apotransférine $10^{-6} \mathrm{M}$ ([HEPES $]=[$ bicarbonate $\left.]=10^{-4} \mathrm{M}-\mathrm{pH}=7,4\right)$.

Les mesures de fluorescence sont effectuées à $20^{\circ} \mathrm{C}$ dans des cuves de quartz de $4 \mathrm{~mL}$. Le $\mathrm{pH}$ des solutions des cuves est mesuré au moyen d'un $\mathrm{pH}$ mètre conventionnel (LPH430T, Tacussel) équipé d'une électrode combinée miniature (MIXC710, Tacussel) reconditionnée par une solution $\mathrm{NaClO}_{4} 0,1 \mathrm{M}$ et $\mathrm{NaCl} 10^{-3} \mathrm{M}$.

\subsubsection{CIEF}

Procédure de greffage du capillaire - Les capillaires de silice vierge $(0,1 \mathrm{~mm}$ de diamètre interne) sont greffés par de l'acrylamide afin d'éliminer l'électroendosmose suivant la méthode suivante (modification de la méthode de Hjertén (1985)) : le capillaire est rincé successivement par de la soude $\mathrm{N}$ (10 min), 
de l'eau ( $2 \mathrm{~min}$ ), une solution à $0,5 \%$ de $\gamma$ methacryloxypropyltrimethoxysilane (Sigma) dans $50 \%$ d'acétone $(20 \mathrm{~min})$ et, finalement, par une solution à $4 \%$ (w/v) d'acrylamide contenant $0,4 \mu \mathrm{L}$ de $\mathrm{N}, \mathrm{N}, \mathrm{N}$ ', N'-tetraethylenediamine (TEMED) et $0,5 \mathrm{mg}$ de persulfate d'ammonium (produits Aldrich - rinçages : haute pression de $20 \mathrm{psi}$ ). Après cette procédure de greffage, le capillaire est rincé par de l'eau puis séché par aspiration.

Étapes de préfocalisation et de mobilisation - Le système P/ACE d'électrophorèse capillaire est placé en polarité inverse. Le capillaire est rempli par l'échantillon mélangé au Bio-Lyte à $2 \%$ durant 1 min (pression 20 psi). L'acide phosphorique $20 \mathrm{mM}$ et la soude $20 \mathrm{mM}$ sont respectivement utilisés en tant qu'anolyte et catholyte. La préfocalisation (première étape) est effectuée durant $7 \mathrm{~min}$ sous une tension de $15 \mathrm{kV}$. Le courant à l'intérieur du capillaire décroît pendant cette période. La mobilisation anodique (seconde étape) des zones focalisées est mise en œuvre en remplaçant l'anolyte acide phosphorique par de la soude $20 \mathrm{mM}$ (tension maintenue à $15 \mathrm{kV}$ ). La migration des zones focalisées est enregistrée à $280 \mathrm{~nm}$ (fenêtre de détection de $100 \times 200$ $\mu \mathrm{m}^{2}$ au niveau de la cartouche contenant le capillaire). La température du capillaire durant les analyses est programmée et maintenue à $25^{\circ} \mathrm{C}$. Entre les différentes analyses, le capillaire est successivement rincé par $\mathrm{HCl} \mathrm{0,1} \mathrm{M}$ et par de l'eau (1 min pour chaque rinçage).

\section{Résultats et discussion}

\section{1. Études en SLRT}

Les études en SLRT ont été effectuées dans quatre milieux différents : bicarbonate, phosphate, citrate et transférine. Ces systèmes représentent les principaux agents complexants présents en milieu sanguin. Bien que de nombreux rapports molaires uranium : ligand aient été analysés, seuls les résultats représentatifs des concentrations des ligands en milieu sanguin sont donnés. Par ailleurs, les résultats du milieu hydroxyle sont utilisés en tant que milieu de référence. Des spectres de fluorescence présentant des variations de $\mathrm{pH}$ à porte de mesure fixe (délai et longueur) sont présentés afin de mettre en évidence les variations spectrales et cela pour chaque système considéré. Seules les modifications spectrales sont considérées dans cette étude. Les mesures de temps de vie des différents complexes présents dans chaque système seront abordés dans des études ultérieures. 


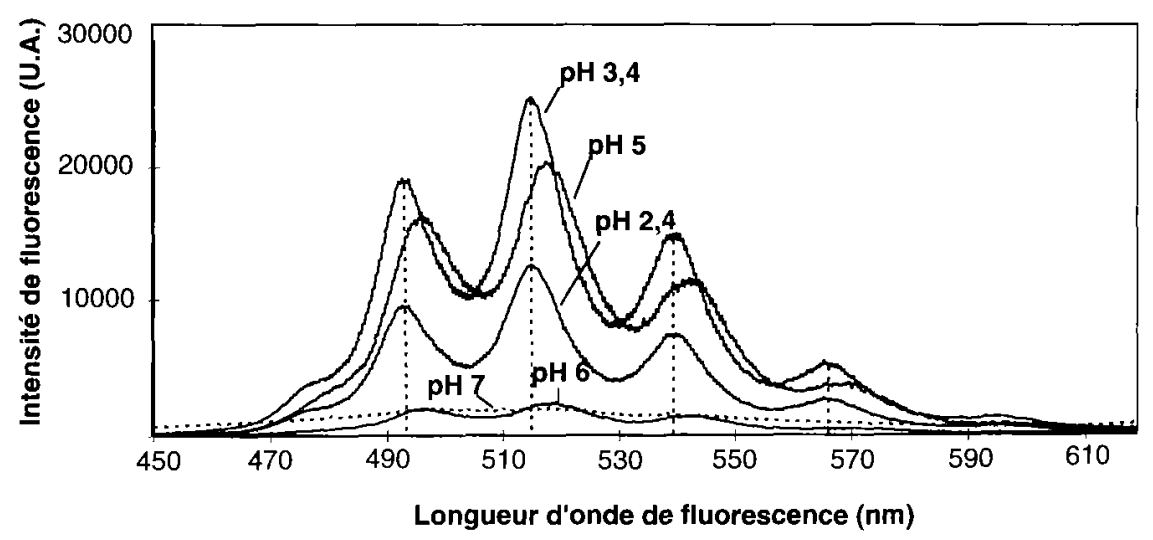

Fig. 1. - Spectres de fluorescence de $\mathrm{UO}_{2}{ }^{2+}$ en fonction du pH - Conditions : $[\mathrm{U}]=2,8 \times 10^{-7} \mathrm{M}, 0, \mathrm{IM}$ $\mathrm{NaClO}_{4}$ - Porte de mesure : $0,5 \mu \mathrm{s}-100 \mu \mathrm{s}$ (délai, longueur) - Temps d'intégration : $1 \mathrm{~s}$ $(p H=7: 5 s)$.

Uranyl fluorescence spectra as a function of pH - Conditions: $[U] 2.8 \times 10^{-7} \mathrm{M}, 0.1 \mathrm{M}$ $\mathrm{NaClO}_{4}$. - Gate delay $0.5 \mu$ and gate length $100 \mu \mathrm{s}$ - Integration time $1 \mathrm{~s}$ (pH 7:5 s).

\subsubsection{Les interactions uranium avec les milieux inorganiques}

Système uranium - $\mathrm{OH}^{-}$- Les spectres de fluorescence des solutions d'uranium VI $\left(2,8 \times 10^{-7} \mathrm{M}\right)$ en fonction du $\mathrm{pH}$ en milieu non complexant $\mathrm{NaClO}_{4}$ $0,1 \mathrm{M}$ sont présentés sur la figure 1 . Ces résultats mettent en évidence plusieurs phénomènes : premièrement, nous remarquons une augmentation de l'intensité de fluorescence jusqu'à $\mathrm{pH}=3,4$; puis une diminution de cette intensité associée à un déplacement bathochrome de $4 \mathrm{~nm}$. En effet, entre $\mathrm{pH}$ 2 et 5 , l'ion uranyle $\mathrm{UO}_{2}^{2+}$ et la molécule $\mathrm{UO}_{2} \mathrm{OH}^{+}$sont présents en solution avec une augmentation de la concentration de cette dernière espèce en fonction du pH (Moulin et al., 1995). A pH = 5, la contribution de l'ion uranyle forme libre devient négligeable en raison des grandes différences en terme de temps de vie entre l'ion uranyle et sa première espèce hydrolysée $\mathrm{UO}_{2} \mathrm{OH}^{+}$ (respectivement 2 et $80 \mu \mathrm{s}$ ). À pH neutre, d'autres espèces telles que les complexes dihydroxo et carbonatés de $\mathrm{UO}_{2}^{2+}$ apparaissent, ce qui explique une diminution de la fluorescence globale. 


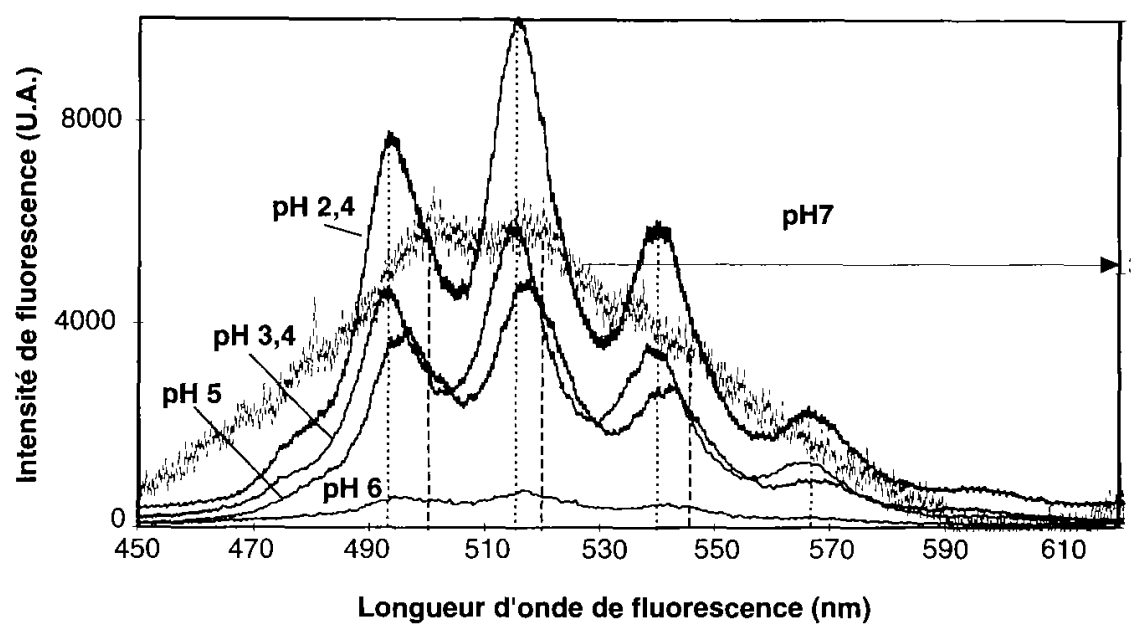

Fig. 2. - Spectres de fluorescence du système Bicarbonate - $\mathrm{UO}_{2}^{2+}$ en fonction du pH-Conditions : $[\mathrm{U}]=2,8 \times 10^{-7} \mathrm{M},\left[\mathrm{NaHCO}_{3}\right]=2,5 \times 10^{-2} \mathrm{M}, 0, \mathrm{I} \mathrm{M} \mathrm{NaClO}{ }_{4}$ - Porte de mesure $: 0,5 \mu \mathrm{s}$ $100 \mu$ s (délai, longueur) - Temps d'intégration : $1 \mathrm{~s}$.

Uranyl - bicarbonate fluorescence spectra as a function of $\mathrm{pH}$ - Conditions: $[\mathrm{U}] 28 \times 10^{-7} \mathrm{M}$, $\left[\mathrm{NaHCO}_{3}\right] 2.5 \times 10^{-2} \mathrm{M}, 0.1 \mathrm{M} \mathrm{NaClO}_{4}$ - Gate delay $0.5 \mu \mathrm{s}$ and gate length $100 \mu \mathrm{s}$ : Integration time $1 \mathrm{~s}$.

Système uranium - bicarbonate - La figure 2 représente les spectres de fluorescence du système uranium - bicarbonate en fonction du $\mathrm{pH}$ de la solution. $\grave{A} \mathrm{pH}$ acide, les différents ajouts de $\mathrm{NaHCO}_{3}$ ne perturbent pas le spectre de fluorescence en raison des réactions suivantes:

$$
\begin{aligned}
& \mathrm{H}^{+}+\mathrm{HCO}_{3}^{-}(\mathrm{aq}) \rightarrow \mathrm{H}_{2} \mathrm{CO}_{2}(\mathrm{aq})\left(\mathrm{K}_{1}=10^{-6,35}\right) \\
& \mathrm{CO}_{2}(\mathrm{~g}) \leftrightarrow \mathrm{CO}_{2}(\mathrm{aq})\left(\mathrm{k}=10^{-1,47}\right) .
\end{aligned}
$$

$\mathrm{Au} \mathrm{pH}=5$, la présence d'une forte concentration de bicarbonate $\left(2,5 \times 10^{-2} \mathrm{M}\right)$ provoque la formation de complexes carbonatés, peu ou pas fluorescents, ce qui induit une diminution de l'intensité de fluorescence globale du système. Un déplacement bathochrome de $6 \mathrm{~nm}$ des principales longueurs d'onde de fluorescence est mis en évidence au $\mathrm{pH}=7$. 


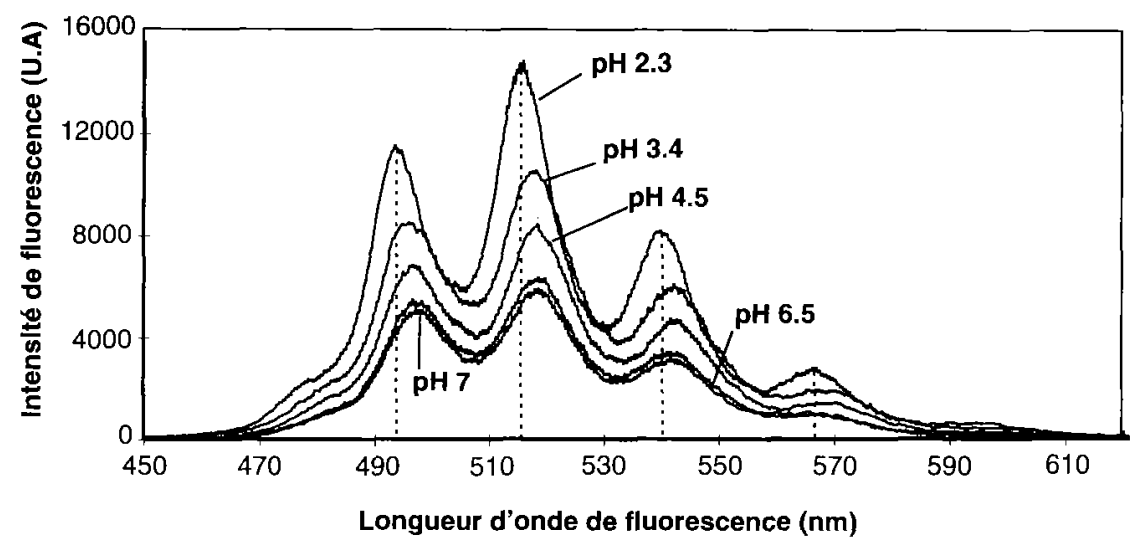

Fig. 3. - Spectres de fluorescence du système Phosphate - UO $\mathrm{O}_{2}^{2+}$ en fonction du pH - Conditions : $[\mathrm{U}]=2,8 \times 10^{-7} \mathrm{M},\left[\mathrm{Na}_{2} \mathrm{HPO}_{4}\right]=1,05 \times 10^{-3} \mathrm{M}, 0,1 \mathrm{M} \mathrm{NaClO} \mathrm{C}_{4}$ - Porte de mesure : $0,5 \mathrm{\mu s}$ $90 \mu s$ (délai, longueur) - Temps d'intégration : $0,5 \mathrm{~s}(\mathrm{pH}=2,3: 1 \mathrm{~s})$.

Uranyl - phosphate fluorescence spectra as a function of pH - Conditions: $[U] 2.8 \times 10^{-7} \mathrm{M}$, $\left[\mathrm{Na}_{2} \mathrm{HPO}_{4}\right] 1.05 \times 10^{-3} \mathrm{M}, 0.1 \mathrm{M} \mathrm{NaClO}_{4}-$ Gate delay $0.5 \mu$ and gate length $90 \mathrm{~ms}$ Integration time $0.5 \mathrm{~s}$ ( $\mathrm{pH} 2.3 \mathrm{~s}$ I s).

Système uranium - phosphate - Concernant le système uranium-phosphate (Fig. 3), à pH = 2,3, les longueurs d'onde de fluorescence (494, 516, 540 et $565 \mathrm{~nm}$ ) caractéristiques du milieu phosphaté $\left(0,75 \mathrm{M} \mathrm{H}_{3} \mathrm{PO}_{4}\right)$ sont observées (Moulin et al., 1995).

Durant les variations de $\mathrm{pH}$ et cela jusqu'au $\mathrm{pH}$ neutre, les longueurs d'onde de fluorescence sont déplacés de $2 \mathrm{~nm}$. Par ailleurs, les intensités de fluorescence diminuent en fonction $\mathrm{du} \mathrm{pH}$ et une modification non négligeable de la largeur à mi-hauteur est remarquée (de 15 à $20 \mathrm{~nm} \mathrm{du} \mathrm{pH} \mathrm{2,3} \mathrm{au} \mathrm{pH} \mathrm{7).}$ En effet, pour ces $\mathrm{pH}$ (et pour des concentrations en uranium de $2,8 \times 10^{-7} \mathrm{M}$ ), les complexes uranium - phosphate présents en solution sont vraisemblablement $\mathrm{UO}_{2} \mathrm{H}_{3} \mathrm{PO}_{4}^{2+}\left(\beta_{1}=10^{0,8}\right)$ et $\mathrm{UO}_{2}\left(\mathrm{H}_{2} \mathrm{PO}_{4}\right)_{2}\left(\beta_{1}=10^{4,8}\right)$ [4] et ils contribuent à une modification des spectres de fluorescence.

\subsubsection{Les interactions uranium avec les milieux organiques}

Système uranium - citrate (voir Fig. 4.) - L'acide citrique $\left(\mathrm{C}_{6} \mathrm{H}_{8} \mathrm{O}_{7}\right)$ présente différents sites de complexation (trois sites carboxyliques et un site hydroxyle) avec différentes constantes d'acidité $\left(\mathrm{pK}_{\mathrm{a}}: 3,1 ; 4,8 ; 6,4\right.$ et 16$)$.

$\mathrm{Au} \mathrm{pH}=2,3$, c'est à dire une valeur inférieure au premier $\mathrm{pK}_{\mathrm{a}}$, seul le spectre de fluorescence correspondant à la première espèce hydrolysée de l'ion uranyle est observé. À pH = 3,4, un élargissement des bandes de fluorescence vers les plus hautes longueurs d'onde est remarqué, phénomène certainement dû à 
la formation de complexes avec les citrates. Dès que le $\mathrm{pH}$ augmente, 1 'intensité de fluorescence diminue en raison probablement d'un quenching statique. Ce phénomène sera confirmé par des mesures de temps de vie de fluorescence.

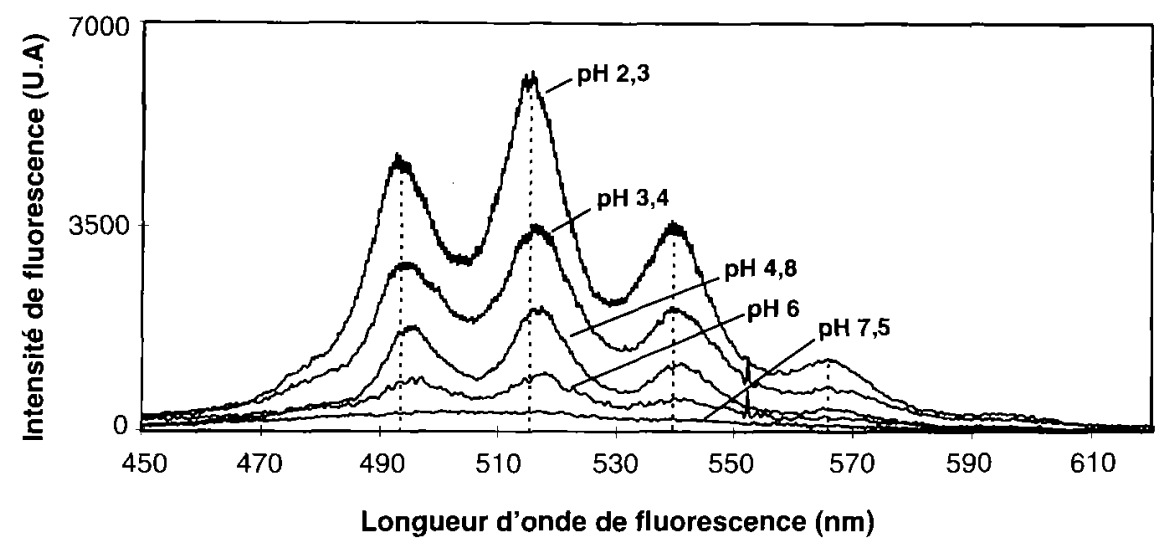

Fig. 4. - Spectres de fluorescence du système Citrate - UO $\mathrm{O}_{2}^{2+}$ en fonction du pH - Conditions : [U] $=2,8 \times 10^{-7} \mathrm{M},\left[\mathrm{C}_{6} \mathrm{H}_{8} \mathrm{O}_{7}\right]=1,59 \times 10^{-4} \mathrm{M}, 0,1 \mathrm{M} \mathrm{NaClO}{ }_{4}$ - Porte de mesure $: 0,5 \mu \mathrm{s}-50 \mu \mathrm{s}$ (délai, longueur) - Temps d'intégration : 1 s.

Uranyl - citrate fluorescence spectra as a function of pH - Conditions: $[\mathrm{U}] 2.8 \times 10^{-7} \mathrm{M}$, $\left[\mathrm{C}_{6} \mathrm{H}_{8} \mathrm{O}_{7}\right] 1.59 \times 10^{-4} \mathrm{M}, 0.1 \mathrm{M} \mathrm{NaClO} \mathrm{O}_{4}$-Gate delay $0.5 \mu$ and gate length $50 \mu \mathrm{s}$ Integration time $1 \mathrm{~s}$.

Système uranium - transférine - En milieu physiologique (tampon HEPES $\mathrm{pH}=7,4)$ en présence d'anions synergistiques tels que les bicarbonates, l'addition d'uranium entraîne une augmentation attendue des intensités de fluorescence.

Plusieurs commentaires peuvent par ailleurs être faits : entre les rapports molaires 0,2 et 2,2 ([U]/[Tf]), un spectre de fluorescence caractéristique des interactions avec la transférine est obtenu comme l'indique la figure 5 ( $[\mathrm{U}] /[\mathrm{Tf}]=2,2)$. Au-dessus de ces rapports molaires, un spectre de fluorescence radicalement différent est observé et identifié au complexe hydroxo $\left(\mathrm{UO}_{2}\right)_{3}(\mathrm{OH})_{5}{ }^{+}$(Laszak et al., à paraître). L'interprétation de ces résultats en terme de constantes de complexation sera l'objet d'une prochaine publication. 


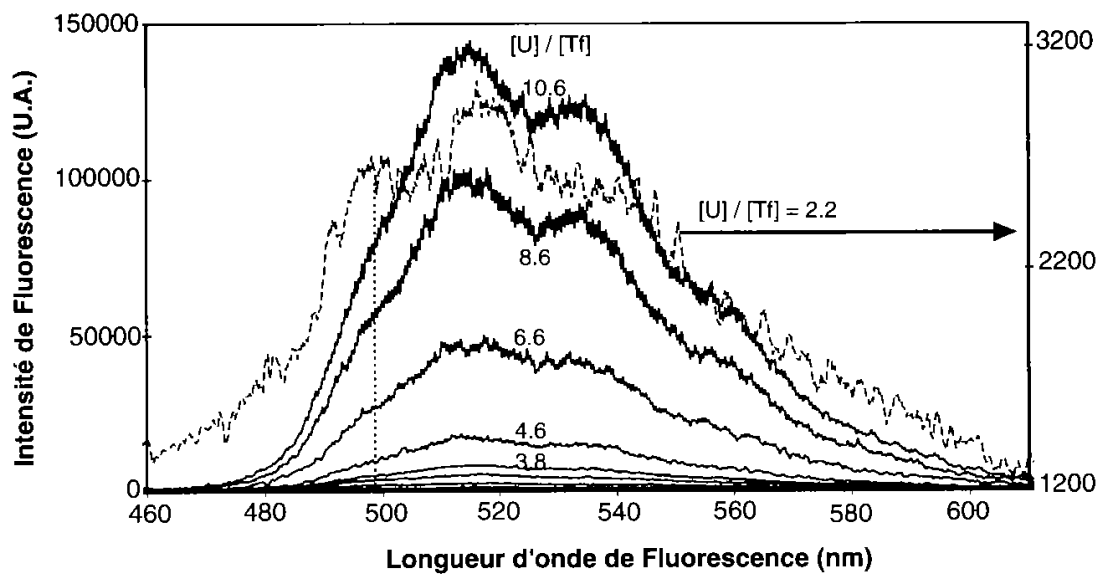

Fig. 5. - Spectres de fluorescence du système Transférine - UO $O_{2}^{2+}$ en fonction de [U]/[Tf]. Conditions : $[\mathrm{Tf}]=10^{-6} \mathrm{M},[\mathrm{Hepes}]=\left[\mathrm{NaHCO}_{3}\right]=10^{-4} \mathrm{M}-$ Ajouts : $[\mathrm{U}]=4 \times 10^{-5} \mathrm{M}$ $(p H=3)[U] /[T f]=0-0,2-0,6-1-1,4-1,8-2,6-3-3,8-4,6-6,6-8,6$ et 10,6-Porte de mesure $: 0,5 \mu s-50 \mu \mathrm{s}$ (délai, longueur) - Temps d'intégration : $10 \mathrm{~s}$.

Uranyl - transferrin fluorescence spectra as a function of [U]/[Transferrin]. Conditions: [Tf] $10^{-6} \mathrm{M}, 10^{-4} \mathrm{M}$ HEPES and $\mathrm{NaHCO}_{3}-\mathrm{pH} 7.4$ - Additions: [U] $4 \times 10^{-5} \mathrm{M}$ pH $3-$ Molar ratios: 0, 0.2, 0.6, 1, 1.4, 1.8, 2.6, 3, 3.8, 4.6, 6.6, 8.6 and 10.6 Gate delay $0.5 \mu \mathrm{s}$ and gate length $300 \mu \mathrm{s}-$ Integration time $10 \mathrm{~s}$.

\section{2. Études en électrophorèse capillaire (mode focalisation iso- électrique - CIEF)}

En CIEF, la clé du développement analytique est d'abaisser l'effet du flux électroosmotique (FEO) afin de stabiliser les zones de focalisation. Pour cela, la procédure de greffage décrite par Hjertén (1992) est appliquée, pour éliminer simultanément le FEO et l'adsorption des protéines sur les groupements silanols composant la surface de silice vierge.

Afin d'obtenir une forte liaison spécifique entre l'uranium et l'apo-transférine, les bicarbonates sont utilisés en tant qu'anions synergistiques (Baker, 1994) et le tampon HEPES demeure la matrice physiologique. 


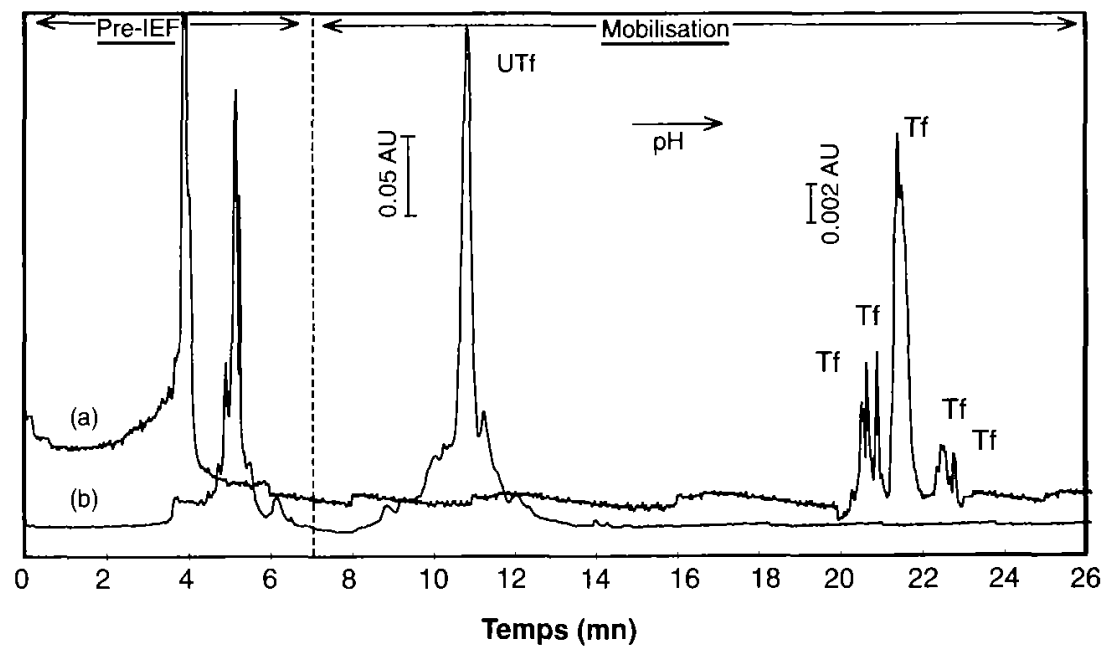

Fig. 6. - Électrophérogrammes de l'apo-transférine (a) et du système uranium-transfêrine (b) par CIEF. Conditions : $[T f]=1 \mathrm{mg} / \mathrm{mL}$ - Electrolyte : Bio-Lyte $5 / 7$ à $2 \%$; anolyte : acide phosphorique $20 \mathrm{mM}$ (pre-IEF) ou soude $20 \mathrm{mM}$ (mobilisation anodique); catholyte : soude $20 \mathrm{mM}$; Détection $U \mathrm{~V}, 280 \mathrm{~nm} ; \mathrm{V}=15 \mathrm{kV} ; \mathrm{T}=25^{\circ} \mathrm{C}$.

High performance isoelectric focusing of free-iron transferrin (a) and uraniumtransferrin (b) samples. Conditions: [Tf] $1 \mathrm{mg} / \mathrm{mL}$ - Electrolyte: $2 \%$ Bio-Lyte 5/7; anolyte: $20 \mathrm{mM}$ phosphoric acid (pre-IEF) or $20 \mathrm{mM}$ sodium hydroxide (anodic mobilization); catholyte: $20 \mathrm{mM}$ sodium hydroxide, on-tube detection at $280 \mathrm{~nm}$, voltage $15 \mathrm{kV}$; temperature $25{ }^{\circ} \mathrm{C}$.

Les électrophérogrammes des analyses par CIEF de l'apo-transférine (spectre (a)) et du système uranium-transférine (spectre (b)) sont représentés sur la figure 6. Comme l'a précédemment décrit Kilar (Kilar et Hertjén, 1989), les pics de la première étape de l'analyse pour chacun des échantillons (Pre-IEF : 0-7 min), correspondent aux liaisons des protéines en mouvement, lesquelles diffusent vers les régions où le $\mathrm{pH}$ est égal à leurs pIs. Dans la seconde étape (mobilisation : 7-26 min), l'accent est porté sur les différences en terme de temps de rétention entre le complexe uranium-transférine, qui apparaît à des temps d'élution autour de 10-12 min, et l'apotransférine (spectre (a)), dont le spectre comporte cinq pics vers 20-23 min correspondant probablement aux formes 2-,3-,4-,5- et 6-sialo de l'apotransférine. Le plus haut pic du spectre caractéristique de la forme 4-sialo est par ailleurs mis en évidence. De plus, on remarque une hauteur de pic significativement plus importante pour le complexe uranium-transférine $(0,26$ U.A.) que pour l'apotransférine $\left(1,4 \times 10^{-2}\right.$ U.A.). Ces deux informations soulignent et confirment la liaison spécifique entre l'uranium et l'apo-transférine. 
Actuellement, d'autres études sont en cours et ont en particulier pour principal objectif d'améliorer les conditions de stabilité de la procédure de greffage du capillaire de silice vierge.

\section{Conclusion}

La spectrofluorimétrie laser à résolution temporelle et l'électrophorèse capillaire utilisée en mode focalisation isoélectrique ont été, pour la première fois, utilisées en tant qu'outil de spéciation en milieu biologique. Les résultats obtenus par SLRT révèlent d'importantes modifications spectrales en fonction du $\mathrm{pH}$ pour les systèmes inorganiques et organiques. Par ailleurs, il s'avère possible de caractériser l'interaction entre l'apotransférine et l'uranium par titration au moyen des spectres de fluorescence. Les mesures des temps de vie des espèces présentes en solution devront permettre de caractériser plus précisément les interactions étudiées. Le mode focalisation isoélectrique utilisée en électrophorèse capillaire (CIEF) a permis, malgré des problèmes de stabilité au niveau du greffage, de mettre en évidence, pour la première fois en EC, la complexation uranium-transférine.

\section{RÉFÉRENCES}

Baker E.N. (1994) Structure and reactivity of transferrins. Advances in Inorganic Chemistry., 41, 389463.

Chevari S. et Likhner D. (1968) Complex formation of natural uranium in the blood. Med. Radiol., 13(8), 53-57.

Couston L., Pouyat D., Moulin C. et Decambox P. (1995) Speciation of uranyl species in nitric acid medium by Time-Resolved Laser-Induced Fluorescence. Appl. Spectrosc., 49(3), 349-353.

Grenthe I., Fuger J., Lemire R., Muller R.J., Nguyen-Trung C. et Wanner H. (1992) Chemical Thermodynamics of Uranium, OECD Nuclear Energy Agency North-Holland, Amsterdan - London - New-York - Tokyo.

Hjerten S. (1985) High-Performance Electrophoresis - Elimination of Electroendosmosis and solute adsorption. J. Chromatogr., 347, 189-198.

Hjerten S. (1992) Isoelectric Focusing in Capillaries, in Capillary Electrophoresis: Theory and Practice, Grossman P.D., Colburn J. C., Academic Press, San Diego, 191-214.

Kilar F. et Hjerten S. (1989) Fast and high resolution analysis of human serum transferrin by high performance isoelectric focusing in capillaries. Electrophoresis, 10, 23-29.

Laszak I., Moulin C., Moulin V. et Tondre C., Appl. Spectro. (à paraître).

Moulin C., Decambox P., Mauchien P. et Moulin V. et Theyssier M. (1991) On the use of Laser-Induced Time-Resolved Spectrofluorometry for interactions studies between organic matter and actinides: application to curium. Radiochim. Acta., 52/53, 119-125.

Moulin C., Decambox P., Moulin V. et Decaillon J. G. (1995) Uranium speciation in solution by TimeResolved Laser-Induced Fluorescence. Anal.Chem., 67, 348-353. 
Moulin C., Reiller P., Beaucaire C. et Lemordant D. (1993) Time-Resolved Laser-Induced Spectrofluorometry for the study of uranium-anionic surfactant micelle interactions. J. Coll. Int. Science., 157, 411-417.

Moulin V., Tits J., Moulin C., Decambox P., Mauchien P. et De Ruty O. (1992) Complexation behaviour of humic substances towards actinides and lanthanides studies by Time-Resolved LaserInduced Spectrofluorometry. Radiochim. Acta., 58/59, 121-128.

Pasquier C. et Bourguignon M. (1977) Thérapeutique des contaminations par I'uranium. CRSSA76, Trav. Scient., 6, 149-154.

Scapolan S., Ansoborlo E., Moulin C. et Madic C., Investigations by Time-Resolved Laser-Induced Fluorescence and Capillary Electrophoresis of the Uranyl-Phosphate Species: Applications to blood serum. J Alloys and Compounds (à paraître).

Scalopan S., Ansoborlo E., Moulin C. et Madic C. Uranium(VI)-transferrin system studied by TimeResolved Laser-Induced Fluorescence. Rad. Prot. Dos. (à paraitre).

Stevens W., Bruenger F., Atherton D.R., Smith J.M. et Taylor G.N. (1972) The distribution and retention of hexavalent ${ }^{233} \mathrm{U}$ in the beagle. In: Actinides in man and animals, Proceedings of the Snowbird Actinide Workshop, october 15-17, M.E. Wrenn ed., 457-472.

Voetglin C., et Hodge H.C. (1953) Pharmacology and toxicology of uranium compounds, Mc GrawHill Book Company, New-York. 